\title{
HISTORIZANDO AL NIÑO AHISTÓRICO DEL CURRÍCULUM PORTUGUÉS DE EDUCACIÓN ARTÍSTICA EN LA ENSEÑANZA PRIMARIA. LA EDUCACIÓN ARTÍSTICA EN LA ESCUELA COMO FORMA DE GOBIERNO DEL ALMA DEL NIÑO
}

\section{Historicizing the Abistorical Child of the Portuguese Primary Arts Education Curriculum. The Arts in School as the Governing of the Child's Soul}

\author{
Catarina S. MARTINS \\ Universidade de Oporto \\ Correo-e: catarina.martins.fbaup@gmail.com
}

Recibido: Io de septiembre de 2020. Envío a informantes: 19 de septiembre de 2020.

Aceptación definitiva: 28 de marzo de 202I

RESUMEN: Esta contribución aborda la escritura de la historia de la educación como una historia del presente. La historia del presente es vista como un estilo de razón concebido, a la vez, como un gesto metodológico y teórico. Busca, a través de un cuestionamiento de las zonas ciegas del presente, rastrear sus orígenes. No se trata simplemente de volver al pasado en busca de la verdad. Por el contrario, lo que la obra de Michel Foucault nos permite es percibir las contingencias, las arbitrariedades, las fragilidades y, tantas veces, la violencia que encierra un conjunto de presupuestos, de formas de decir, de ver y de pensar que utilizamos en el ámbito educativo. De forma más concreta, me centraré en el currículo de la educación artística básica en Portugal, para mostrar cómo se construye no a partir de una relación con las artes, sino a través de sucesivas capas históricas en las que las representaciones del niño, de los métodos y de los procesos de aprendizaje se alimentan de los lenguajes psicopedagógicos con el objetivo de gobernar quién debe ser el niño y también quién no debe ser ese niño. Thomas Popkewitz se refiere a estas alquimias curriculares como el proceso de traducción que transforma un determinado conocimiento en una materia curricular, según las retículas de racionalidad procedentes de la psicología. La educación artística no tiene que ver con las artes, sino que es 
una traducción de los principios psicológicos del niño a la escuela. Con este proceso alquímico vienen también las reglas de cómo se supone que los niños aprenden, qué etapas del desarrollo deben atravesar, cómo se debe dividir y proporcionar el conocimiento y cómo las artes reflejan la vida interior del niño. La producción de conocimiento psicopedagógico sobre el niño produciría imágenes sobre lo que el niño es y sobre lo que debería llegar a ser.

El gobierno de la infancia encuentra sus líneas genealógicas en el proceso de gobierno del cuerpo social, en el que al poder de gobernar correspondería siempre un gesto minucioso de producción de conocimiento sobre los gobernados. Así, una mirada a los currículos desde este enfoque teórico nos permite analizar no solo la estructura de la escuela, sino también las gramáticas que la ponen en funcionamiento, y problematizar el niño ahistórico del currículo de educación artística como un proyecto mayor que tiene en el gobierno del niño (y simultáneamente del maestro, de los padres, de las familias, de la sociedad) su finalidad. Hoy, los lenguajes adquieren diferentes matices, las categorías se multiplican, pero la racionalidad presentada en los currículos de educación artística sigue siendo la misma.

PALABRAS ClAVE: currículum portugués; educación artística; escuela primaria.

AвSTRACT: The contribution addresses the writing of the history of education as a history of the present. The history of the present is seen as a style of reason conceived, both, as a methodological and theoretical gesture. It seeks, through a questioning of blind zones in the present, to trace its origins. It is not simply a question of returning to the past in search of truth. On the contrary, what Michel Foucault's work allows us is to perceive the contingencies, arbitrariness, fragilities and, so often, violence enclosed in a set of presuppositions, ways of saying, seeing and thinking that we do act in the educational field. In a more concrete way, I will focus on the basic arts education curriculum in Portugal, to show how these are constructed not from a relationship with the arts, but through successive historical layers in which representations of the child, of the methods and processes of learning feed on the psychopedagogical languages with the aim of governing who the child should be and also who should not be that child. Thomas Popkewitz refers to these curricular alchemies as the translation process that transforms a given knowledge into a curricular subject, according to grids of rationality coming from psychology. Arts education is not about the arts but is a translation of psychological principles of the child into schooling. With this alchemical process there also come the rules of how children are supposed to learn, which stages of development they are supposed to go through, how knowledge should be divided and provided, and how the arts mirror the child's inner life. The production of psychopedagogical knowledge about the child would produce images about what the child is and about what it should become.

The government of childhood finds its genealogical lines in the process of governing the social body, in which to the power of governing would always correspond a meticulous gesture of knowledge production about those to be governed. Thus, a look at the curricula from this theoretical approach allows us to analyze not only the structure of the school, but also the grammars that put it into operation, and to problematize the ahistorical child of the arts education curriculum 

CATARINA S. MARTINS

as a larger project that has in the government of the child (and simultaneously the teacher, parents, families, society) their purpose. Today, languages acquire different nuances, the categories multiply, but the rationality presented in the arts education curricula remains the same.

KEY WORDS: Portuguese curriculum; arts education; primary school.

\section{Introducción}

$\Theta^{\text {I }}$

I OBSERVAMOS cómo se contempla al niño en los discursos educativos, tenemos la impresión de encontrarnos ante un niño que siempre ha existido, y que nunca dejará de serlo así hasta alcanzar la edad adulta. Esta idea del niño como ser en desarrollo no solo ha arraigado en las formas de pensar, hablar y actuar sobre la infancia, sino que también su fragilidad, maleabilidad, curiosidad, creatividad, entre otras, parecen estar siempre vinculadas a una forma natural de ser niño. Entonces se plantea una pregunta sobre cómo comprender hoy esa racionalidad que convierte al niño y a la infancia en un acontecimiento histórico.

La primera parte de este artículo aborda la forma de escribir la historia de la educación como una historia del presente (Foucault, I980 [1971]). La historia del presente es comprendida desde un tipo de razón, como un gesto metodológico y teórico (Popkewitz, 2013) para rastrear su límite en el presente cuando se pregunta por las zonas ciegas. No se trata simplemente de volver al pasado en busca de la verdad. Por el contrario, el trabajo de Michel Foucault nos permite percibir los límites, la arbitrariedad, las fragilidades y, con frecuencia, la violencia incluida en un conjunto de presuposiciones, formas de decir, ver y pensar en el presente, desde las que actuamos en el ámbito educativo.

La pregunta que hacemos es ¿cómo llega a ser posible pensar y actuar con la infancia como lo hacemos nosotros? La respuesta a esta pregunta presupone una noción muy específica del poder y del conocimiento en la producción de los sistemas de razón que construyen el niño y, simultáneamente, organizan la reflexión y la participación. El gobierno de la niñez encuentra sus líneas genealógicas en el proceso de gobierno de la población, en que el poder de gobernar correspondería siempre a un gesto meticuloso de la producción de conocimiento sobre todos aquellos que van a ser gobernados (Foucault, I982). Este conocimiento adquirió formas específicas, al mismo tiempo, dando forma a aquello que estaba siendo objeto de observación. La infancia, por ejemplo, podría ser estudiada estadísticamente a través de las tasas de mortalidad o los registros de enfermedad y, psicológicamente, mediante indicadores de desarrollo o de coeficiente intelectual (Turmel, 2008). Por lo tanto, el conocimiento es una consecuencia del poder.

El camino seguido en este artículo sitúa al poder en una posición muy específica. Desde la modernidad el poder se ejerce cada vez menos mediante la fuerza bruta y más a través de formas de conocer quiénes somos y deberíamos ser, y que provocan las prácticas de autogobierno. Michel Foucault (I99I) llamó a esta forma 
de razonar gubernamentalidad. La gubernamentalidad hereda formas de gobierno que proceden de un poder pastoral. Sin embargo, ya no se relaciona con la salvación de las almas para una vida posterior, sino con la secularización del alma, en términos de crear el ciudadano moderno y el gobierno de los hombres. La noción de poder como provechoso es importante para desplazar las nociones habituales de poder como algo represivo. La construcción de la ciudadanía se basaba en el ejercicio autónomo de la conducta por cada sujeto. El gobierno liberal, por tanto, no se opone a la libertad del sujeto. Al contrario, la libertad es un instrumento esencial para el gobierno liberal para organizar las condiciones bajo las que cada ciudadano se hace y puede hacer uso de su libertad.

Desde fines del siglo XIX en adelante, la noción de libertad también aparece como un a priori cuando vamos a hablar de artes y la creación del artista moderno. Esto será importante si tenemos en cuenta las vías por las que las artes llegan a la escuela y cómo se han establecido relaciones estrechas entre el artista moderno y la imagen de niño y de infancia. En el mundo del arte, el siglo XIX se identifica como un desafío en la forma que los artistas se imaginaban a sí mismos. La academia, vista como conservadora, fue cuestionada y el acto de criticar las reglas de la academia apareció como un acto de libertad. A fines de siglo el ensamblaje de la noción de "arte primitivo», los «nuevos» artefactos no occidentales traídos a Europa y llevados a Estados Unidos bajo la etiqueta de «arte», las nociones de evolución de las especies y los dibujos infantiles también abrieron otras vías para la expansión de los significados de la libertad de los artistas y para la consideración de los dibujos infantiles como una cierta verdad sobre el niño y su desarrollo que la psicología se encargaría de traducir (Martins, 20I7). A comienzos del siglo XX muchos artistas hablaron de la necesidad de desaprender para lograr un estado infantil «puro». El «desaprendizaje» encontró su equivalente en la escuela, en la idea de «libre expresión». Esta naturalización de la libertad de expresión como parte de las formas en que se expresan las artes en la escuela se abordará en la segunda parte del artículo.

En la sección tercera observaré cómo, dentro del currículum de las artes, esta libertad como autoexpresión y los enfoques motivadores para aprender y enseñar las artes forman parte del gobierno del niño que activa técnicas confesionales, en las que las artes se convierten en una tecnología de la vigilancia de sí mismo (Martins, 20I4). Por tanto, una mirada a los currículos desde este enfoque teórico nos permite problematizar el niño ahistórico del currículum de educación artística como un proyecto más amplio que busca el gobierno del niño.

Cuando hablo de las artes en el currículum de la escuela primaria me refiero a las artes visuales, música, teatro y educación física, que se denominan «expresiones artísticas». Incluso si se tratan todos estos diferentes medios «artísticos», resulta difícil encontrar las conexiones existentes entre ellos y las prácticas artísticas. La cuarta parte se centrará en cómo el currículum de educación artística se construye no desde la relación entre las artes, sino mediante sucesivas capas históricas en las que las representaciones del niño, y de los métodos y procesos de enseñanza, se alimentan de los lenguajes psicopedagógicos que buscan gobernar 

CATARINA S. MARTINS

quién debe ser el niño y quién no debe ser ese niño. Thomas Popkewitz (2007) se refiere a estas alquimias curriculares como el proceso de traducción que transforma un conocimiento dado en un tema curricular, de acuerdo con las leyes de racionalidad que proceden de la psicología. La educación artística no trata de las artes, sino que es una traducción de los principios psicológicos que organizan quién es el niño y cómo debería estar en la escuela. Junto con estos procesos de alquimia vienen las reglas de cómo se supone que los niños deben aprender, qué etapas de desarrollo se supone que han de atravesar, cómo debe dividirse y administrarse el conocimiento y cómo las artes reflejan la vida interior del niño (Martins, 20I8). Cuando es examinada con estas lentes, la educación artística queda vinculada con la representación del ciudadano deseado para el futuro, pero no con las artes.

\section{La configuración del niño como un evento}

En esta sección en primer lugar situaré la historia del presente como un modelo teórico y metodológico de hacer historia. Para participar en este sentido de historia, es necesario repensar y revisar cómo se entiende el pasado en el presente. La historización implica una contextualización que mira al presente tratando de percibir en él la emergencia de un conjunto de naturalizaciones. La diferente toma de posición entre historicismo y hacer historia se señala como algo importante en la relación que ha de establecerse con el archivo. El historicismo considera los documentos del archivo como elementos informativos que abren la posibilidad de explicar una evolución temporal de los hechos que sucedieron en el pasado. Desde la perspectiva de historización, estos documentos son percibidos como «acontecimientos» que ordenan los objetos de reflexión y participación en un determinado tiempo y espacio. Lo que viene a ser importante no es describir cómo se producen los hechos, sino cómo pudieron ocurrir así. Estas condiciones de posibilidad son las que dan razón de ser a lo que el historicismo considera como realidad. La pregunta es pensar sobre los principios que definen lo que cuenta como objeto de estudio. Si nosotros consideramos la noción de niño o de infancia como fenómenos históricos, entonces tendremos que desnaturalizar la forma en que vemos y actuamos hoy con los niños, entendiendo en primer lugar que son consecuencias del poder. La segunda parte de esta sección intenta hacer este desmontaje, mostrando cómo las reglas y los estándares de razonamiento sobre el niño son construcciones sociales y culturales. Lejos de mirar al niño como una entidad fija, proponemos considerar la emergencia moderna del niño como parte de la nueva racionalidad del gobierno en la modernidad.

\section{I. Una ontología del presente}

Cuando uno lee el currículum portugués de educación artística para la escuela primaria, una cosa parece casi evidente: el currículum «conoce» al niño sobre el 
que se está hablando de tal manera que llega a ser casi imposible para el lector cuestionar las suposiciones que se están haciendo. Estas suposiciones no solo se relacionan con formas particulares de ver, pensar y actuar sobre el niño mediante una racionalidad cronológica y evolutiva, sino también, por ejemplo, cuál es el tamaño y la medida de los contenidos adecuados para cada edad y grado; el mejor proceso para hacer que el niño aprenda, o las mejores estrategias para hacer más atractivo y efectivo el proceso de enseñanza y aprendizaje. Una forma de observar las representaciones contenidas en el discurso del currículum es considerar que se relacionan con un conocimiento que se basa empíricamente y corresponde a una verdad sobre la naturaleza del niño y su desarrollo, por ejemplo. De hecho, esta es la forma más habitual de mirar y relacionarse con el currículum, y, sin embargo, este es un efecto de poder.

Desde el siglo XIX, la construcción científica del niño, a los ojos de los médicos, psicólogos y educadores, generó un conocimiento que define cómo vemos al niño, pero al mismo tiempo también cómo se abre la infancia como un campo de actuación en términos de corrección o incremento del desarrollo del cuerpo infantil o su inteligencia. Gráficos y cuadros fueron instrumentos que, por ejemplo, registrando el crecimiento o la madurez del cuerpo y la inteligencia traducían una idea de desarrollo y mediaban la relación entre niños, padres, educadores y diferentes instituciones. Los instrumentos para captar qué se había entendido como crecimiento del niño cambiaron, pero uno de los más influyentes entre ellos fue el dibujo. Si observamos, por ejemplo, las formas por las que el niño es examinado a través de sus dibujos y es inscrito en ciertos estadios de desarrollo de acuerdo con él, vemos que hay formas de razonar que se naturalizaron en el siglo XIX cuando se recopilaron y compararon baterías de dibujos infantiles. De estas colecciones surgieron formas de ver al niño normal y su Otro, siendo este el niño que siguió un camino diferente desde el punto de vista de su expresión gráfica.

El enfoque utilizado en este artículo es considerar que cada discurso no solo es una forma de hablar sobre temas, objetos o el mundo, sino sobre todo la fabricación de los temas, objetos y del mundo de una cierta forma. Si pensamos, de nuevo, en los dibujos de los niños, hemos de preguntarnos cómo fue posible pensar sobre ellos en términos de desarrollo biológico y psicológico infantil, y mediante este razonamiento considerar su desarrollo como normal, por debajo o encima de la norma. Esta forma de relacionarse con el currículum de educación artística tiene en cuenta la política del conocimiento que hace que el discurso del currículum sea un hecho que ordena la reflexión y la participación. Por ejemplo, si a los siete años el niño dibuja o pinta como se espera que lo haga a la edad de tres o si los contenidos del dibujo son considerados como inapropiados para el niño, la mirada de los educadores señalará el «hecho» y de ahí pueden seguirse diferentes actuaciones. En este sentido, analizar lo que da razonabilidad a las formas de pensar, hablando y actuando en relación con el niño, implica una relación particular con lo que implican las prácticas del discurso. Tomo prestado de Michel Foucault esta noción de discurso no como 
una tenue superficie de contacto, o confrontación, entre una realidad y un lenguaje (lengua), la imbricación de un léxico y una experiencia; [...] Una tarea que consiste en no -ya no- tratar los discursos como grupos de signos (elementos significativos que se refieren a contenidos o representaciones), sino como prácticas que de forma sistemática dan forma a los objetos de los que hablan. (Foucault, 1972, pp. $48,49)$

Las prácticas del discurso no son algo que pertenece al momento del ahora, sino que son el resultado de una serie de acumulaciones históricas que «suceden» en el presente. Esto es lo que básicamente opone historicismo e historización. En cierto sentido, ello permite mirar el currículum de educación artística, y al niño en él representado, no como un tema surgido en la historia. Como argumenta Popkewitz, «donde paradójicamente el historicismo organiza el pasado para hablar del futuro, la historización es un proyecto crítico para hacer frágil la aparente causalidad del presente» (2013, p. 2). Reconocer el discurso del currículum como un hecho nunca busca adecuarlo a una supuesta realidad, sino más bien comprender sus propias condiciones de vida y articulación y, por tanto, desnaturalizar lo que se da por supuesto, ya sea el niño visto como un aprendiz, como un ser creativo o como un ser en desarrollo.

Una ontología del presente, una historia del presente o una genealogía se relacionan con una actualización crítica de la pregunta filosófica de Kant sobre "¿qué es la Aufklarung?». La pregunta histórica de Michel Foucault implicaba esta estrecha conexión con lo que está sucediendo ahora y qué es «ahora» lo que nos permite ver, pensar y actuar de una forma determinada. En una entrevista, declaró con claridad que deseaba

centrarse en lo que estaba sucediéndonos hoy, qué somos, qué es nuestra sociedad. Pienso que nuestra sociedad y lo que somos tiene una profunda dimensión histórica, y en este espacio histórico los hechos del discurso que han sucedido hace siglos o años, son muy importantes. Nosotros estamos entretejidos en el interior de esos acontecimientos del discurso. De alguna forma no somos nada más que lo dicho. (Foucault, 1978 [1976], pp. Io, II)

El estudio seguirá tratando de hacer evidente cómo el niño que aparece en el currículum de la educación artística es construido históricamente y cómo esto se relaciona con prácticas de gobernar al niño y transformarlo en ciudadano a través de la escuela.

\subsection{Eliminar al niño abistórico del discurso educativo: el gobierno del niño}

En los discursos renovadores hoy la pregunta parece ser cómo encontrar formas más efectivas de conectar con los modos en que los niños «realmente aprenden». Esta pregunta se expresa de diferentes formas: cómo motivar a los niños, qué contenidos son los más cercanos a lo que es específico de la cultura contemporánea 


\section{HISTORIZANDO AL NIÑO AHISTÓRICO DEL CURRÍCULUM PORTUGUÉS DE EDUCACIÓN ARTÍSTICA EN LA ENSEÑANZA 228 PRIMARIA. LA EDUCACIÓN ARTÍSTICA EN LA ESCUELA COMO FORMA DE GOBIERNO DEL ALMA DEL NIÑO CATARINA S. MARTINS}

de los niños, cómo transformar la forma aburrida de conocer en materiales estimulantes, cómo equilibrar el tiempo que el niño debe prestar atención con el tiempo de juego, cómo utilizar más tecnología en el aula o, al contrario, como imaginar una clase más abierta a la naturaleza o al parque infantil, entre otras vías. Ninguno de estos temas es nuevo en términos educativos. Si retrocedemos un siglo nos daremos cuenta de cómo la pedagogía progresiva siempre ha pensado cómo adaptarse al niño, respetando su «naturaleza». Pero esta naturaleza infantil se construye bajo la mirada de la ciencia; se materializa a través de redes de prácticas, y tiene, desde sus inicios, propósitos bien definidos de gobierno. Si preguntamos, por ejemplo, cómo se piensa que el niño es un «niño en desarrollo», nos damos cuenta de que la noción de desarrollo define al niño normal y su Otro. Lo cual permite incluir ciertos niños y alimenta prácticas excluyentes. Lo que la historización de ciertos tropos sobre lo que es natural para el niño o para la infancia puede ofrecernos la posibilidad de ver cómo estos han sido elaborados y abiertos al cálculo y la administración.

Desde el siglo Xvil, sobre todo, es cuando comienza a consolidarse la moderna conceptualización de la infancia. No es que antes el niño no fuera visible en el día a día de la comunidad, sin embargo, el niño aparecía como una versión del adulto en pequeño, y la infancia era un tiempo breve, pronto sustituido por su incorporación al trabajo. Philippe Ariès (1962), por ejemplo, muestra cómo, en muestras visuales, el niño aparecía representado no por su especificidad (entendida con los ojos de la modernidad), sino bajo la apariencia de adulto. La vida estaba dividida en «edades», pero estas no solo correspondían a las fases biológicas, sino a funciones sociales y tenían una relación directa con el cosmos. En el siglo XVI en Francia se imprimió una compilación latina del siglo xiII con el título Le Grand Propriétaire de Toutes les Choses. Si abrimos el libro sexto de esta «enciclopedia» encontramos las edades de la vida, que se dice que son siete y se corresponden con los planetas. La primera edad era la infancia, y duraba siete años; seguida de la pueritia, hasta los catorce, y la adolescencia, que duraba hasta los veintiocho años. La descripción de cada una de estas edades es irreconocible para la mente y los ojos de hoy. No hay características psicológicas o de desarrollo de los niños, no hay interés por su porvenir, sino solo un conjunto de guías que constituyen el significado de la vida del hombre en su relación con el mundo. Un mundo antropocéntrico, un mundo hecho con relatos de prodigios, maravillas, signos, analogías o cifras, que constituyen el gobierno de Dios sobre el hombre, es sustituido durante los siglos XVI y XVII. Michel Foucault (2007, pp. 312, 313) llamó al cambio en que la naturaleza y el hombre fueron vistos y representados la des-gubernamentalización del cosmos y, al mismo tiempo, la gubernamentalización del Estado.

El nuevo concepto de niño e infancia forma parte de la nueva forma de pensar sobre los sujetos en términos de cómo ellos forman parte de y se integran en un cuerpo de población que constituye la nación, y cómo este niño llega a ser ciudadano. Una vía teórica y metodológica de comprender esta nueva forma de pensar es mediante la gubernamentalidad (Foucault, 199I). Gubernamentalidad 

CATARINA S. MARTINS

fue el neologismo que inventó Foucault para referirse a la nueva racionalidad del gobierno desde la modernidad. El gobierno era comprendido por medio de un conjunto de principios intrínsecos al propio Estado, y no procedentes de Dios, lo que significaba que la idea de gobierno podría no verse como algo contrario a los asuntos o sujetos gobernados. Foucault se refería a un nuevo arte de ordenar y dirigir la conducta de cada ciudadano, cada vez más como una tarea que cada cual se impondría a sí mismo en lugar de servirse de la fuerza o la coerción. «Designaba la forma en que podría ser dirigida la conducta de los individuos o de los grupos: el gobierno de los niños, de las almas, de las comunidades, de las familias, de los enfermos» (Foucault, 1982, p. 22I). Sustentado en esta racionalidad de gobierno existiría un territorio, la seguridad de ese territorio, la felicidad de sus habitantes, desde entonces comprendidos como una población. Este tipo de gobierno sería biopolítico, situando la vida como su centro. Y en el centro de esta clase de gobierno estaría la necesidad de elaborar un conocimiento capaz de hacer efectivo un gesto de poder y, simultáneamente, hacer que este poder del conocimiento forme parte de las prácticas habituales de las instituciones, haciendo familiares estos procedimientos y ampliándolos a la sociedad en general, en particular extendiendo su alcance a espacios como la familia, desde la intimidad a la gestión de la casa, desde la alimentación a la crianza del niño.

Lo particular de la gubernamentalidad es combinar la herencia de un poder pastoral con la conducta del rebaño, mediante la idea de la salvación del alma, con la economía política del gobierno de los hombres, basada en un meticuloso conocimiento de la vida, que podría establecer zonas de peligro que merecen una intervención directa en el sentido de su corrección o control. En la modernidad, la salvación del alma adopta diferentes significados: salud, felicidad, bienestar, seguridad, educación, entre otros.

Una de las principales preocupaciones de la gubernamentalidad era la escuela pública y la educación. No solo era una nueva forma de colocar niños dentro de una nueva institución llamada escuela, sino más bien proponer una nueva concepción de lo que era un niño y debería ser como un ser moral y un ciudadano perteneciente a la familia y la comunidad. La educación llevaba consigo una narrativa de salvación, de progreso y ascenso a la vez que representaba la posibilidad de diferenciar y gobernar al niño, la familia y su relación con la comunidad. Como expone Popkewitz, esta trilogía vincula al niño con la «naturaleza» de la familia y de la comunidad. La educación moderna y sus prácticas escolares han funcionado como una forma de construir individuos, mediante la etiqueta de normalidad y la administración de su libertad. La autorrealización individual «estaba ligada a la pertenencia colectiva para mejorar el bien común» (Popkewitz, 2008, p. 79).

Las ciencias de la educación, como parte de la gubernamentalidad y organizadas como un nuevo campo de estudio, aportaron tesis culturales (Popkewitz, 2008) sobre cómo criar al niño de acuerdo con su desarrollo y atributos naturales (Martins, 20I7). Sin embargo, el nuevo niño moderno era distinto, por ejemplo, del niño de Rousseau. En Rousseau el niño era «bueno» por naturaleza, su mente era vulnerable y necesitada de la protección del mundo adulto, y por ello el 
niño debería ser dejado libre para que siguiera el orden de la naturaleza de su propio desarrollo. Rousseau es fundamental para comprender la emergencia de un nuevo espacio para pensar sobre el niño, y educadores como Pestalozzi y Froebel partieron de sus ideas, pero un enfoque diferente del niño fue el que impulsó el movimiento del Child Study en los Estados Unidos y en Europa. La educación progresiva en Estados Unidos, y también en Europa, consideraba el estudio científico de los niños como su misión. Las mismas técnicas estadísticas utilizadas para el estudio científico de las poblaciones se aplicaron y desarrollaron en el estudio del niño, en particular para saber cómo se desarrolla el niño, aportando nociones sobre normalidad y desviación. La ciencia era la clave para ordenar, clasificar y planificar la vida social, que incluía la transformación de la gente mediante la educación. Las formas científicas de ver y decir naturalizaron categorías específicas de la infancia a partir de una relación entre el niño normal y el anormal.

Cuando, por ejemplo, en 1883 G. Stanley Hall publica The Contents of Children's Minds, surge un nuevo tipo de niño. Este niño estaba siendo elaborado desde la mirada del psicólogo, mediante la aplicación de tests al grupo, mediante la observación y una forma de razonamiento comparativo. Fue entonces cuando se desarrollaron los tests usando el dibujo como un instrumento para acceder y evaluar cómo se había desarrollado la interioridad del niño, confirmando la idea de etapas del desarrollo, basadas en el progreso lineal. No era la naturaleza la que debía gobernar al niño, sino que el niño debería regirse por el conocimiento de su naturaleza.

A finales del siglo XIX, el pedagogo francés Gabriel Compayré afirmaba que la ciencia de la educación era una ciencia práctica, ni más ni menos que una psicología aplicada. No era el único que afirmaba esto y las leyes de la pedagogía fácilmente se volvieron inseparables de un conocimiento psicológico meticuloso de quienes iban a ser educados. A comienzos del siglo Xx esta idea se repitió en Portugal como una urgencia.

El pedagogo portugués António Aurélio da Costa Ferreira, por ejemplo, escribió que «el arte de educar» sería fundamentalmente «el arte de regular la conducta presente y futura de los educandos [...] Educar es condicionar de forma intencional las reacciones del individuo». Sin embargo, afirmar esto significaba dominar la vida interior del niño, para que el maestro, como un pastor, pudiera intervenir directamente en su alma: «Trabajar el alma del niño con el alma y con el arte, con el arte y con la corrección, con la corrección y con la ciencia, está a nuestro alcance» (Ferreira, 1919, pp. 17-20).

La construcción del niño bajo las lentes de la psicología y la pedagogía desde entonces ha estado asociada a las prácticas de gobierno. Las materias de la escuela, así como sus contenidos y la racionalidad que les da sentido, entonces no pueden verse como derivados de su esencia (lo que es el niño), sino de un conjunto de conocimientos y prácticas que dan racionalidad a cómo debemos pensar que es el niño, y que tienen propósitos de gobierno. Por ejemplo, se introdujeron las artes en el ámbito educativo portugués dentro de una dinámica de moralización de la 

CATARINA S. MARTINS

población, dirigida de forma particular hacia los niños y los jóvenes «en peligro» (Martins, 2019). Lo que estaba en juego era una comprensión de las artes como una tecnología capaz de transformar, desde el interior, a quienes las practicaban. Por tanto, las artes se asociaban con propósitos y principios gubernamentales basados en prácticas de autogobierno.

\section{Las artes como instrumentos para la expresión del sí mismo íntimo}

Cuando analizamos el discurso curricular artístico para la educación primaria en Portugal, las artes aparecen a través de una retórica de efectos (Gaztambide-Fernández, 20I3; Martins, 20I8). Las artes, se dice, permiten a los niños expresarse libremente siendo creativos, y esta libertad se entiende como opuesta a cualquier coerción del profesor y como un hecho completamente positivo. El programa de artes visuales establece que «manipular y experimentar con materiales, formas y colores permite a los niños desarrollar formas personales de expresar su mundo interior y representar la realidad mediante descubrimientos sensoriales» (Educação, n. d., p. 89). La pregunta que surge es, ¿qué subyace a la idea de las artes como instrumentos de expresividad y cómo es posible pensar en las artes como vehículos de expresión del yo interior? Para responder a esto, uno debe mirar primero cómo se estableció un concepto moderno del yo y cómo ha llegado a naturalizarse en el discurso de las artes y en el de la educación artística.

Comprender una persona a través de la noción del yo forma parte del fenómeno de la modernidad. En el periodo grecorromano, conocerse a uno mismo para cuidar de uno mismo era una práctica que se tenía, por ejemplo, como un mecanismo para acceder a la verdad. Sin embargo, este conocimiento de uno mismo que buscaba hacer de la vida un arte de vivir es sustantivamente distinto de la relación cristiana con el yo, como anota Foucault.

Por ejemplo, Roy Baumeister (1987) se sirve de la literatura para captar las diferencias que desde la baja Edad Media han ido conformando la manera en que una persona observaba y organizaba su vida en función de su concepto del yo. Si en la época medieval moralidad y virtud formaban dos de los atributos que daban sentido a la vida de uno, desde el siglo XIX en adelante el yo se convierte en un problema de personalidad como identidad y la realización de uno se comenzó a articular con la individualidad, imaginación, la singularidad personal, la pasión y la emoción. Estos avances forman parte de la emergencia de la psicología como un campo científico. La psicología concibe el yo como algo oculto que podría ser explicado a través de ciertas acciones que daban visibilidad al yo. Se habló del yo a través de la noción de personalidad, convirtiéndose en objeto de búsqueda, observación y medicalización. La narrativa biográfica que convierte en ficción una historia de vida, en casos clínicos, legales o artísticos, es considerada como la vena de acceso a la primera y más profunda de las causas que dan sentido a las actuaciones futuras de los sujetos, ya sean locos, criminales o genios. En el arte, como 
en la literatura, la obra producida fue recibida mediante el concepto del autor, y se utilizó esa misma obra para comprender a la persona que la creó.

Esta persona era el artista, pero un artista diferente al del Renacimiento o el Barroco. El régimen vocacional de las artes, que floreció durante el siglo XIX, yuxtapone la noción protestante de vocación a la noción romántica de genio como la que dicta las reglas de la naturaleza, y la noción psicológica del yo a través de la singularidad, la individualidad y la personalidad. Durante el siglo XIX, aunque las academias de arte crecieron en número, este crecimiento se vio acompañado de la devaluación del aprendizaje académico en favor de la libertad y el individualismo del genio. Aunque tomados como caminos opuestos, las academias artísticas y el artista moderno se alimentaron de la misma tecnología de gobierno: la del genio. Dentro de la academia, las antiguas galerías de los genios eran vistas como las que dictaban las reglas y el lugar a que cada estudiante debía aspirar. Los que se oponían a la rigidez de la escuela se consideraban, ellos mismos, los verdaderos genios. Esta última posición se basaba en la idea de libertad de expresión y en la noción que ser artista era más una cuestión de naturaleza que de educación (Ó, Martins y Paz, 2013).

La cuestión de la libertad, la expresión de los sentimientos, la consolidación de la individualidad y la originalidad se convierten en los tropos que separan al artista moderno de sus primeras versiones. La excepcionalidad del genio se erige en norma mediante la noción de singularidad. Lo biográfico se afirma como una tecnología de gobierno mediante la normalización de lo singular. Esta forma de razonamiento era la propia de la gubernamentalidad mediante el uso de la libertad y la individualidad como instrumentos de autogobierno.

Estas confrontaciones se trasladaron a la arena pedagógica. La batalla entre un dibujo de expresión libre y un dibujo de carácter más riguroso y geométrico se oponía a las posiciones sobre la enseñanza de las artes y de cómo el niño estaba siendo conceptualizado en la escuela. Terminó ganando la perspectiva libre, basada en el mito romántico del artista que lucha contra la idea de la escuela y el aprendizaje, y que se acerca a una forma de purismo mediante el examen de sí mismo. Encontró en la perspectiva evolucionista una recapitulación de las especies que conduciría al niño de los garabatos hasta un dibujo más realista y menos imaginativo. Esta perspectiva libre también formaba parte de las formas en que el arte primitivo y las obras de «arte» y «cultura» no occidentales estaban siendo apropiadas por el mundo del arte moderno.

Clifford (1988) muestra cómo objetos procedentes de fuentes no occidentales se dividían en dos grandes categorías: artefactos culturales o bien obras de arte. A comienzos del siglo Xx muchas obras de arte revelaron semejanzas con, por ejemplo, los objetos africanos, lo que se percibía como la búsqueda de una especie de purismo o verdad interior que aparentemente unía a tres figuras: 'salvajes' o 'primitivos', artistas y niños.

En la década de i88o psicólogos y artistas se fijaron en producciones infantiles, los primeros tratando de observar y representar científicamente qué era el niño; los segundos se sintieron asombrados por las cualidades estéticas y semejanzas 

CATARINA S. MARTINS

existentes entre el arte de los niños y el arte primitivo, que se percibía como una especie de elemento puro que pronto sería corrompido por la sociedad. James Sully, uno de los nombres más emblemáticos en el estudio del dibujo infantil, argumenta que la primera expresión tosca del sentido estético del niño tenía puntos en común con «el gusto por correr» Sully, 1890, p. 299). Se apostaba por el estudio científico del niño, y se afirmaba que el niño pasaba por varias etapas de desarrollo que se correspondían con el progreso de la raza humana. El autor de Studies of Childhood destacó que «el deleite en el brillo de las cosas relucientes, en los tonos alegres, en los fuertes contrastes de color», se aproximaba al «primitivo y la mirada pueril era del gusto del hombre civilizado» (Sully, I890). También se fijó con sentido común que la expresión libre del artista se aplicaba a la conceptualización de la infancia, y viceversa. El intercambio entre las dos figuras resultó ser provechoso. Faria de Vasconcelos, un pedagogo portugués, no tuvo dudas al afirmar que,

sin una plasticidad vigorosa o capacidad de cambiar y renovarse, sin una curiosidad continua y animosa, sin el poder de la fantasía y la imaginación creativa, sin el gusto por el juego, que es ensayo y riesgo, sin la visión sencilla y directa que excluye toda rutina, sin el estado de gracia, entusiasmo y alegría, sin el don de sí mismo que se entrega con optimismo y confianza, sin la fe que actúa y la acción que va directamente a su objetivo, no hay artistas, ni sabios. Estas cualidades son las de la infancia, el principio de la vida y la condición del progreso. (Vasconcelos, 20I0-1934, p. 13)

Si se aceptó como un a priori que el arte era la expresión visible y manifiesta de una interioridad, se aceptó que el mismo yo fuera un objeto de observación para conocer mejor y dominar esa interioridad. La psicología se sirvió de diversas herramientas para estudiar y construir la personalidad del niño, cómo se suponía que debía aprender y desarrollarse y, con este fin, se adaptaron varios tests utilizando imágenes y dibujos. La idea del desarrollo infantil, como un proceso en que lo biológico se codifica en una escala de atributos y capacidades, encuentra en el dibujo su propia posibilidad de extenderse. Se utilizó el dibujo para acceder al alma del niño y hacerle consciente de esta «alma».

A partir de i88o, en el seno del Movimiento Internacional del Estudio del Niño, las etapas del desarrollo mediante el dibujo señalizaron la biologización del desarrollo. El dibujo aportó pruebas para que fueran asumidas como datos objetivos sobre cómo el niño, y la misma especie humana, se desarrolló en el horizonte del tiempo. La relación directa entre edad y rendimiento se transformó en preocupación por convertirse en un tema político y moral para el gobierno del niño.

El estudio de la naturaleza infantil mediante el dibujo hizo que el tiempo importara como un instrumento para regular el progreso del niño a través de diferentes etapas, y se consideraron los dibujos como esenciales para apreciar la naturaleza del niño. Se utilizaba el dibujo con instrumento metodológico para recopilar datos empíricos y luego como instrumento de diagnóstico, señalando 
los elementos peligrosos que había dentro de un grupo de población, pero también como un instrumento de regulación desde el que se medía la normalidad del niño y como espejo de comparación entre niños diferentes de la misma edad cronológica. El dibujo fue instrumentalizado como un espejo del interior del alma infantil, distinguiendo entre quién era el niño y cómo debería desarrollarse. Cyril Burt, un psicólogo inglés, llegó a afirmar que los dibujos infantiles «abren entradas a lugares extraños de la mente infantil, zonas que de otra manera permanecerían intactas y sin explorar» (1922, p. 317).

Las ciencias de la educación después de la II Guerra Mundial y el discurso sobre educación artística se fijaron en el niño como un ser creativo por naturaleza que debía ser apoyado para que mejorara su potencial. Las actividades libres, expresivas y creativas se consideraron equivalentes a tareas relacionadas con el arte, siendo los padres y los maestros los únicos responsables de proporcionar a los niños el entorno y objetos adecuados que podrían transformarlos en adultos democráticos, saludables, flexibles y creativos (Martins, 2020). El educador del arte Viktor Lowenfeld, en una guía para padres sobre el niño y su arte, subrayó la idea de que «el arte para su hijo podría significar el equilibrio necesario para el intelecto y las emociones de su niño» (I954, p. 9).

Las actividades de expresión no solo tendrían un fin terapéutico para regular el intelecto y las emociones infantiles, sino sobre todo consecuencias morales y de gobierno para conseguir un equilibrio mientras se libera la imaginación, y facilitar rutas para conocer al niño. El niño aprendería cómo ser consciente de sí mismo y la adecuada dimensión de este equilibrio.

En esta primera sección he tratado de hacer una breve contextualización para reflexionar sobre la presencia de las artes en la escuela como una actividad propia de la infancia y como libre expresión. Leyendo el currículum de educación artística se puede ver que «la interpretación libre de los significados de la expresión gráfica y plástica» contribuirá «a despertar la creación y creatividad de los estudiantes» (Ministerio da Educação, p. 89). Estas llamadas a universalizar la articulación entre las artes y el niño son ahistóricas pues ignoran las prácticas muy específicas culturales que tienen en cuenta al niño o la noción del «arte por el arte». El niño ahistórico es presentado en el currículum de las artes desde una perspectiva de autoexpresión, que retoma las nociones de gobierno en términos de tecnologías del autogobierno o, en otras palabras, hacer de uno mismo el espacio de la intervención y la administración. No solo se coloca al niño en un dispositivo para producir conocimiento sobre sí mismo, sino que se le incita a prácticas de autogobierno. En la siguiente sección, mi objetivo es centrarme en cómo estas formas de imaginar las artes como un espacio de libertad en la escuela se relacionan con técnicas confesionales que convierten el alma del niño en un espacio para la intervención y la administración. 

CATARINA S. MARTINS

\section{Las artes como técnicas confesionales}

Las formas de hablar y asociar las expresiones artísticas a territorios de libertad, en que el niño es libre de expresar sus sentimientos e interioridad y, al mismo tiempo, como una manifestación de su desarrollo, forman parte de las naturalizaciones debatidas más atrás en relación a la constitución del artista moderno y las concepciones del desarrollo infantil que ha producido la psicología. Sin embargo, como vamos a revisar en esta sección, la exploración de su interioridad es una práctica del gobierno que recupera y desarrolla tecnologías confesionales.

Ahora en la escuela primaria se invita y anima a los niños a desplegar su imaginación a través del dibujo y la pintura. La justificación de esta práctica permanece casi oculta para el profesor. Sin embargo, con esta actuación, los niños se transforman en objetos abiertos al cálculo, la evaluación, el escrutinio y la comparación. Al mismo tiempo, se ponen en juego prácticas de subjetividad para comprender que los niños construyen para sí mismos algún tipo de sujetos con ciertas cualidades y capacidades. Las elecciones de cada niño están directamente ligadas a sus sensibilidades. Es deseable, por ejemplo, «que los niños escojan los materiales y colores que mejor se adapten a su sensibilidad» (M. Educação, p. 92). La naturalidad de la interioridad infantil se advierte también a través de otros componentes como los que permite la oportunidad del contacto con la naturaleza, se dice, para «desarrollar la sensibilidad estética del niño» (M. Educação, p. 89). Existen estratos de experiencia psicológica en las formas de imaginar que las artes son capaces de retirarse del fondo de una verdad infantil.

Este concepto de las artes como instrumentos de expresividad construye, dentro de la clase, mecanismos de confesión que activan técnicas de autogobierno. El niño se hace visible como un cierto tipo de niño mediante el acto mismo de la revelación. Como la palabra o la escritura, se comprende el dibujo de forma muy diferente de lo que significa para el niño. La universalización del lenguaje psicológico produce una descontextualización del acto de dibujar, al considerarlo como un espejo de la conducta esperada. En este sentido, al mismo tiempo que el niño es invitado a expresar su interioridad, esta se transforma en el objeto de observación y confirmación del conocimiento general de lo que es el niño. El enfoque se sitúa entonces en la traducción y correspondencia entre las acciones y las representaciones.

El mecanismo de la confesión es, para Foucault, «un ritual de revelación en el que el sujeto que habla es también el sujeto que declara» (1998, p. 6I). En el caso de las artes de expresión, el mecanismo de confesión establecido no es otro que el de la hoja en blanco. El estudiante expresa su imaginación dibujando o pintando y está sometido a la mirada del profesor. «El profesor observará, sin interferir de forma expresiva, cómo utilizan los niños el espacio de pintar: cómo cogen el pincel, rellenan las superficies, utilizan el color, y también perciben el ambiente creado» (M. Educação, p. 93). Cada una de estas acciones crea imágenes sobre el tipo de niño que se va moldeando en el aula bajo las gafas pedagógicas del profesor. Aunque no se indica de forma explícita, el análisis de los modos de aplicar el 
color, de la composición visual o de los temas dibujados desvela imágenes de lo que es el niño, de lo que debería ser, o de los posibles caminos de corrección a emplear con cada niño. «Si los niños muestran más iniciativa, entonces el maestro puede sugerir otras experiencias que profundizarán en la capacidad de los estudiantes para expresarse mediante la pintura» (M. Educação, p. 93). Para ser autónomo, el niño ha de ser conducido por diferentes caminos. No solo se utilizan las artes como instrumentos para desvelar la interioridad, sino que también se utilizan para crear un autoconocimiento mediante la expresión de los sentimientos y pensamientos y, al mismo tiempo, se moldea al niño desde su interior.

El alma del niño se convierte en el foco del gobierno mediante tecnologías de observación e intervención. El niño en proceso de desarrollo está siendo observado a través de un conjunto de hechos que se hacen equivalentes a los talentos naturales del niño. La iniciativa de desarrollo, por ejemplo, se va construyendo mediante diferentes aparatos que hacen visible al niño a través de la continuidad del desarrollo desde experiencias simples a las más complejas. Como argumenta Walkerdine, «la psicología evolutiva y la pedagogía centrada en el niño forman una pareja: los aparatos de la pedagogía no son mera aplicación sino un lugar de producción por derecho propio» (p. 157). El lenguaje de la psicología, para hablar del niño, establece un tipo de conocimiento universal sobre lo que es la infancia y cómo se produce el desarrollo, creando, de esta forma, el niño normal que parece carecer de historia.

Los niños de los que se habla en el currículum de artes son los que se desarrollan de forma natural y aprenden por sí mismos. En el nivel del currículum de música, por ejemplo, «se intenta que el niño sea capaz, por sí mismo o en grupo, de desarrollar sus propios proyectos, contando con la ayuda del profesor en la elección y manejo de los medios utilizados» (M. Educação, p. 72). Situado en el centro, el niño aprende a valorar su propia conducta y a progresar mediante el desarrollo y sus relaciones con los demás. El análisis de los mecanismos de la razón que originan lo que trata de representar el conocimiento y el niño que aprende representan la división entre tipos de niños y cómo se comportan estos niños dentro y fuera del aula. El profesor actúa como el pastor que conduce al niño a la salvación. Esta salvación es el propio desarrollo del niño de acuerdo con las imágenes que emite el niño normal. El dispositivo pedagógico, al activar las tecnologías confesionales, funciona como una caja moral.

\section{Las alquimias de la educación artística: ¿quién se desarrolla mediante las artes?}

Cuando miramos por las lentes de la historia del presente, el programa curricular de las expresiones artísticas nos da un conjunto de a priori sobre cómo aprenden y se desarrollan los niños, y queda claro que la idea de arte que da nombre a la asignatura en la escuela es solamente una etiqueta. Disciplinar y gobernar el propio cuerpo, los gestos, los movimientos, la voz, trabajar con finura 

CATARINA S. MARTINS

las destrezas motrices, resolver problemas y desarrollar habilidades sociales son algunos de los objetivos que impregnan el discurso del currículum. Las formas de pensar sobre la educación artística son semejantes a los principios que trasladan las matemáticas o las ciencias al interior de la escuela. Los debates actuales sobre el currículum de la educación artística, al igual que sucede en otros campos disciplinarios, tienden a proyectarse sobre cuáles son los contenidos adecuados o cómo la enseñanza de las artes pueda ser más eficaz y eficiente. Lo que no se pregunta es qué hace que un determinado contenido pueda ser enseñado y cuáles son las condiciones que hacen razonable al verdadero acto de transformar un conocimiento dado en una porción que pueda ser aprendida por los estudiantes, y al mismo tiempo proporciona un conjunto de «efectos secundarios», como el de transformar a los estudiantes en sujetos autónomos o capaces de resolver problemas.

La traducción que se produce cuando el conocimiento específico de un tema viaja al interior de la escuela está mediatizada por los estratos del discurso psicológico. El nombre no solo es un rótulo, sino que debajo del mismo existen diferentes formas de pensar sobre el desarrollo del niño, los procesos de aprendizaje y la solución de problemas estructurados en capas cuyo objetivo es cambiar y transformar al niño en un cierto tipo de persona. Thomas Popkewitz llama a esto la alquimia de las asignaturas de la escuela. «las asignaturas de la escuela, argumenta, tienen poco que ver con la red y las relaciones que forman y ordenan las normas de participación, verdad y reconocimiento en los diferentes campos académicos vinculados a las asignaturas de la escuela» (Popkewitz, 2007, p. 78). Las alquimias tienen un efecto rector.

Si imaginamos el discurso del currículum como un acontecimiento, podemos tomar los momentos históricos y los ingredientes que lo configuran y lo concluyen como producto terminado en la actualidad. Utilizar la analogía de la tarta puede sernos útil aquí (Martins y Popkewitz, 2015). Vista en una vitrina, la tarta es un objeto determinado. La tarta puede tener muchas propiedades que la hacen deseable: el olor, el color, la textura, el sabor. Mirar la tarta, incluso sin degustarla, puede permitirnos identificar qué tipo de tarta está ante nuestros ojos. Sin embargo, los diferentes ingredientes que la componen se ocultan cuando vemos la tarta sacada del horno. Cada uno de estos ingredientes posee diferentes cualidades, capacidades y características. Por tanto, si observamos el currículum de educación artística como una tarta, necesitamos identificar las diferentes actuaciones que lo hacen posible como un producto terminado, y pensar sobre diferentes ingredientes como si tuvieran propiedades que «hacen» cosas a la gente, en otras palabras, que forman diferentes tipos de personas (Hacking, 2006).

La fabricación de diferentes tipos de personas es visible, por ejemplo, en las formas como se construye la voz como espacio de administración e intervención. En las clases de teatro y música, por ejemplo, los niños tienen que aprender a usar su voz. La voz no es solo la voz. La voz se convierte en el lugar para adaptarse al mundo exterior y las relaciones que se espera desarrollar en el futuro. La variación de la voz, el control de la respiración, el volumen del habla o el rit- 
mo de las palabras deben servir junto a los temas apropiados a la edad del niño, para mostrar que la voz sirve de instrumento de «comunicación y expresión» (M. Educação, p. 79). Aunque la voz, en el campo de la música, se considera natural para el niño, el niño puede tener problemas con su propia naturaleza, pues habrá algunos que encuentren dificultades en el desarrollo de un cierto tipo de música. Sin embargo, se invitará al niño a aprender el control de su voz, del cuerpo y los instrumentos musicales como «un todo», y se le pedirá que «los utilice de forma integrada, armoniosa y creativa» (M. Educação, p. 68). No se explica qué significa una forma integrada, armoniosa y creativa, pero esto no solo destaca una concepción occidental de la música como una expresión artística, con escalas armónicas que corresponden a un cierto tipo de música, sino que también presupone un sujeto que aprende a tener control sobre su propio cuerpo, sus movimientos, sus emociones o sus expresiones faciales. Gritar o permanecer en silencio, por ejemplo, correspondería a un uso de la voz no integrado, inarmónico y no creativo.

Se dice que el desarrollo de la musicalidad debe ser gradual, de acuerdo con las etapas del desarrollo del niño, pero todavía dependiente «del dominio de las habilidades instrumentales, el lenguaje adecuado, el gusto por la exploración, la capacidad de escuchar» (M. Educação, p. 68). La educación artística se traduce en un conjunto de especificidades que marca lo que el niño debe demostrar para estar de acuerdo con la norma. Se presentan estas características como capacidades naturales del niño: habilidades instrumentales, gusto por explorar o la capacidad de escuchar. El niño se hace disponible como un lugar para intervenir y gobernar a través de un entrenamiento más intensivo o mediante la exposición a ejercicios que moldean un cuerpo musical. Las capacidades que posee el niño, o no, crean distinciones entre los cuerpos. Por ejemplo, se afirma que la escucha «es un proceso evolutivo personal y complejo dependiendo de la sensibilidad y la experiencia» (M. Educação, p. 68). El niño tiene la responsabilidad de trabajar en su cuerpo para adquirir la necesaria musicalidad que le permite ser un buen oyente. El discurso de la educación artística incluye y, al mismo tiempo, excluye a quienes no pueden o no se exponen a experimentar la escucha musical.

Pronto nos daremos cuenta de que no estamos hablando del cuerpo musical, sino de un cuerpo para el futuro deseado y disciplinado. El currículum pierde sus articulaciones con las especificidades relacionadas con la música o con otro campo artístico y se convierte en una máquina de civilidad. Las psicologías expresadas en el texto son sistemas de gobierno que sitúan a cada niño en comparación con los demás y dentro de un dispositivo de relaciones sociales reguladas. Aún hay algunos conceptos vinculados al mundo del arte, como el sonido, el ritmo, la notación de sistemas musicales, pero estos se transforman mediante prácticas pedagógicas en aisladas y particulares tareas que el niño con un cierto grado de desarrollo y grado de madurez debe completar. Mediante estas tareas las subjetividades de los niños se van ordenando y regulando de acuerdo a su pertenencia a una zona de la sociedad. Por ejemplo, en la danza y la gimnasia se dice que la interacción entre los niños concuerda con la necesidad de su desarrollo social. El currículum establece principios que forman al ciudadano razonable. Lo que 
podemos observar en el currículum de la educación artística es que los contenidos de la música, el teatro o las artes visuales se configuran a través de un vocabulario psicológico sobre el niño y cómo se supone que el niño debe aprender, primando el desarrollo personal, el control de sí y de su cuerpo, el sentido de pertenencia o las habilidades sociales que el niño tiene que dominar.

\section{Para finalizar, aunque no es el final de la «historia»}

Este texto tiene como objetivo problematizar al niño de que se habla en el discurso circular de la educación artística de la escuela primaria en Portugal. Mi interés ha sido puntualizar los momentos históricos que han construido este niño y, con ello, proponer una forma de pensar sobre la educación de los niños. La historia del presente sirvió, aquí, al mismo tiempo de demostración teórica y metodológica para acceder al presente como un acontecimiento. La eventualidad de los discursos de la educación artística, y lo observado en el niño que vive en ella implica preguntarse sobre el efecto de las prácticas históricas y las relaciones de poder en la construcción del niño como un ciudadano.

Cuando observamos los discursos sobre el currículum de educación artística en la escuela primaria, aparece un niño que parece carecer de historia: es un cierto tipo de niño que aprende de una forma determinada y que se desarrolla de acuerdo con una línea cronológica específica. Esta forma de pensar que el niño tiene una historia y su historia se articula con prácticas específicas de gobierno propias de una racionalidad moderna. Estas prácticas se caracterizaban, sobre todo, por el desarrollo de la individualidad de cada sujeto, mientras, al mismo tiempo, esta individualidad solamente lograba sentido cuando era aceptada por la población. No obstante, en la modernidad el poder nunca marchó solo. El conocimiento es constitutivo de las relaciones de poder y, en el caso de la educación, el conocimiento psicológico sobre el niño generó ciertas aspiraciones de verdad sobre lo que era el niño.

En la escuela, se ha formado la individualidad de los niños mediante prácticas de autogobierno que parecen apelar a la libertad de expresión. En el nivel de la educación artística, esta libertad encuentra su equivalente en la idea, por ejemplo, de un dibujo libre, expresivo y creativo. Sin embargo, en el momento mismo de ser libre y expresivo, los niños están siendo domesticados por anteojeras psicológicas que construyen y predicen qué significa ser libre o expresivo. He intentado desmontar estos discursos mostrando cómo ellos construyen dispositivos confesionales en la clase y cómo activan tecnologías de autogobierno. En cierta forma, mi objetivo era puntualizar que lo que se dice del niño en el currículum de educación artística tiene sus propias condiciones de posibilidad que dan razón de ser a las actuaciones practicadas en la clase. En escasas ocasiones se cuestiona esta razonabilidad, al considerarse que es producto de la observación empírica. Sin embargo, desde una historia del presente, lo visto como «empírico» no puede separarse de las prácticas del discurso histórico que integran los «hechos» que 
pueden ser observados de forma «empírica». Por otra parte, también he tratado de mostrar que las artes de que hablamos en la escuela tienen poco que ver con las artes y sus guerras en el mundo actual. Hay una alquimia que atraviesa los discursos del currículum que se articula con el moldeamiento de un cierto tipo de niño y con su manejo, mucho más que con el desarrollo de un conocimiento específico de las artes, tal como este conocimiento se practica en su respectivo campo disciplinar.

\section{Bibliografía}

ArIÈs, P.: Centuries of Childhood. A social History of Family Life, New York, Alfred A. Knopf, 1962.

Baumeister, R.: «How the Self Became a Problem: A Psychological Review of Historical Research", Journal of Personality and Social Psychology, 52(I) (1987), pp. 163-176.

Clifford, J.: The Predicament of Culture. Twentieth-Century, Ethnography, Literature, and Art, Cambridge and London, Harvard University Press, 1988.

Educação, M. da: I I $^{\circ}$ Ciclo do Ensino Básico. Organização Curricular e Programas. Expressão e Educação: Físico-Motora, Musical, Dramática e Plástica, n. d.

Ferreira, A. A. d. C.: A Arte de Educar e a Psicologia Experimental, Coimbra, Imprensa da Universidade, I9I9.

Foucault, M.: The Archeology of Knowledge and the Discourse on Language, New York, Pantheon Books, 1972.

Foucault, M.: «Dialogue on Power. Michel Foucault and a group of students Los Angeles», en Wade. S. (ed.): Chez Foucault, Los Angeles, Circabook, 1978 [1976], pp. 4-22.

Foucault, M.: «Nietzsche, Genealogy, History», en Bouchard, D. F. (ed.): Language, Counter-Memory, Practice. Selected Essays and Interviews by Michel Foucault, New York, Cornell University Press, 1980 [1971], pp. 139-164.

Foucault, M.: «The Subject and Power», en Dreyfus, H. L. y Rabinow, P. (eds.): Michel Foucault. Beyond Structuralism and Hermeneutics, 1982, pp. 208-226.

Foucault, M.: «Technologies of the Self», en Martin, L. H.; Gutman, H. y Hutton, P. H. (eds.): Technologies Of The Self. A Seminar With Michel Foucault, Massachusetts, The University of Massachusetts Press, 1988, pp. 16-49.

Foucault, M.: «Governmentality», en Burchell, G.; Gordon, C. y Miller, P. (eds.): The Foucault Effect. Studies In Governmentality With Two Lectures By And An Interview With Michel Foucault, Chicago, The University of Chicago Press, I991, pp. 87-I04.

GAZTAMBIDE-FERnÁNDEZ, R.: «Why the arts don't do anything: toward a new vision for cultural production in education", Harvard Educational Review, 83(I) (20I3), pp. 2II-236. Lowenfeld, V.: Your Child and His Art. A Guide for Parents, New York, The Macmillan Company, 1954.

Martins, C. S.: «The Arts in Education as Police Technologies. Governing the Child's Soul», European Education, 45(3) (2014), pp. 67-84.

Martins, C. S.: «From Scribbles to Details: The Invention of Stages of Development in Drawing and the Government of the Child», en Popkewitz, T. S.; Díaz, J. y Kirchgasler, C. (eds.): A Political Sociology of Educational Knowledge. Studies of Exclusions and Difference, New York and London: Routledge, 2017, pp. I05-II8.

Martins, C. S.: «The Alchemies of the Arts in Education. Problematizing Some of the Ingredients of the Recipe», en Jörissen, B.; Klepacki, L.; Klepacki, T.; Flasche, V.; Engel, J. y 
HISTORIZANDO AL NIÑO AHISTÓRICO DEL CURRÍCULUM PORTUGUÉS DE EDUCACIÓN ARTÍ́STICA EN LA ENSEÑANZA PRIMARIA. LA EDUCACIÓN ARTÍ́STICA EN LA ESCUELA COMO FORMA DE GOBIERNO DEL ALMA DEL NIÑO 24I CATARINA S. MARTINS

Unterberg, L. (eds.): Spectra of Transformation, Munster and New York, Waxmann, 20I8, pp. $4 \mathrm{I}-57$.

MarTins, C. S.: «Arts Education in Portugal in the Eighteenth and Nineteenth Centuries: Drawing and the Governing of the Student», en Baldacchino, J.; Freedman, K.; Hall, E. y Meager, Nigel (eds.): The International Encyclopedia of Art and Design Education, Wiley-Blackwell, 2019.

Martins, C. S.: «Post-World War Two Psychology, Education and the Creative Child: Fabricating Differences», en Popkewitz, T. S.; PetTersson, D. y Hsiao, K.-J. (eds.): The International Emergence of Educational Sciences in the Post-World War Two Years Quantification, Visualization, and Making Kinds of People, New York, Routledge, 2020, pp. 9I-Io8.

Martins, C. S. y Popkewitz, T. S.: «The 'Eventualizing’ of Arts Education», Sisyphus - Journal of Education, 3(I) (2015), pp. 7-17.

Ó, J. R. do; Martins, C. S. y PAz, A. L.: «Genealogy as history: From Pupil to Artist as the Dynamics of Genius, Status, and Inventiveness in Art Education in Portugal», en POPKEWITZ, T. S. (ed.): Rethinking the History of Education. Transnational Perspectives on Its Questions, Methods, and Knowledge, New York, Palgrave Macmillan, 2013, pp. 157-178.

PopkewiTZ, T. S.: «Alchemies and Governing: Or, questions about the questions we ask», Educational Philosophy and Theory, 39 (2007), pp. 64-83.

Popkewitz, T. S.: Cosmopolitanism and the Age of School Reform. Science, Education, and Making Society by Making the Child, New York and London: Routledge, 2008.

Popkewitz, T. S.: «Styles of Reason: Historicism, Historicizing, and the History of Education», en Popkewitz, T. S. (ed.): Rethinking the History of Education. Transnational Perspectives on Its Questions, Methods, and Knowledge, New York, Palgrave Macmillan, 2013, pp. I-26.

Turmel, A.: A Historical Sociology of Childhood. Developmental Thinking, Categorization and Graphic Visualization, Cambridge, Cambridge University Press, 2008.

(Traducción de Elena Patricia Hernández Rivero, Universidad de Salamanca) 
